\title{
A Hybrid Feature Selection Algorithm Based on Information Gain and Sequential Forward Floating Search ${ }^{\oplus}$
}

Jianli Ding, Liyang Fu

School of Computer Science and Technology

Civil Aviation University of China

China

jianliding@aliyun.com, liyang_fu@126.com

\begin{abstract}
As an important pre-processing method in machine learning, feature selection eliminates data redundancy and reduces feature dimensions and computational time complexity effectively. In order to further reduce the number of iterations of the feature selection algorithm and improve the classification accuracy, a new hybrid feature selection algorithm is proposed, which combines the filter algorithm based on information gain and the wrapper algorithm based on Sequential Forward Floating Search (SFFS) and Decision Tree (DT). The optimal candidate feature subset is quickly found by ranking the features using information gain. In order to avoid the nesting effect of features, SFFS algorithm is used to reduce feature dimensions of the optimal candidate feature subset. The experiments show that the maximum ratio of the number of reduced features and the number of initial features is $92.86 \%$. Compared with other feature selection algorithms, the maximum decline of the number of iterations is about $67.8 \%$, and the maximum increase of the classification accuracy is about $10.5 \%$. The results prove that the hybrid algorithm possesses higher computational efficiency and classification accuracy.
\end{abstract}

Keywords: Machine Learning, Feature Selection, Information Gain, SFFS, Decision Tree

Received: 26 March 2018, Revised 4 May 2018, Accepted 13 May 2018

DOI:

(C) 2018 DLINE. All Rights Reserved

\section{Introduction}

With the rapid development of computer technology, the accumulation of data grows exponentially. However, valuable information is rare, so how to extract useful information and models from a large number of data quickly and efficiently becomes urgent demands for people. Data mining is an interdisciplinary technology that combines databases, machine learning, pattern recogni-

${ }^{1}$ Supported by Major projects of Civil Aviation Technology Innovation Funds of China (MHRD20150107, MHRD20160109), Tianjin, Key Lab Open Fund for Advanced Signal Processing Civil Aviation University of China (2015ASP02) and Fundamental Research Funds for the Central Universities of Civil Aviation University of China (3122016A001, 3122015C020).

${ }^{2}$ To whom correspondence should be addressed. E-mail: liyang_fu@126.com 
tion, statistics and other researches. The technology extracts potential and useful information from a large number of data and has gradually become one of the most popular methods in the field of data analysis[1]. In order to take advantage of data mining tools efficiently, data preprocessing plays a crucial role. Feature selection is one of the important and common techniques in data preprocessing. It reduces the number of features by removing redundant or irrelevant data, and improves the speed of calculation and prediction accuracy of data mining algorithm[2]. Feature selection is a process of searching for the optimal subset from the initial feature dimension. The typical feature selection process is divided into four main steps: subset generation, subset evaluation, stopping criterion and result validation[3]. Firstly, The subset generation is essentially a process of heuristic search. And it is determined by a search starting point and a search method. The data set with $\mathrm{N}$ feature dimensions contains $2^{\mathrm{N}}$ candidate subsets, indicating that the search space is exponentially related to the feature dimension. Therefore, in order to reduce the computational complexity of the search process, the search method is divided into three strategies: complete search, sequential search and random search. Secondly, the candidate feature subset generated during every search process needs to be evaluated by the evaluation criterion. If a new subset performs better, the previous best subset is replaced by it. The process of subset generation and subset evaluation is repeated until the stop criterion takes effect. Finally, the selected candidate subset needs to be validated and analyzed.

For different evaluation criterions, feature selection algorithms are broadly divided into three categories: Filter Model, Wrapper Model and Hybrid Model[2]. 1) The filter model is independent of the mining algorithm, depending on the general traits of the data to evaluate. Two kinds of filter feature selection methods based on ReliefF and information gain were proposed in [4] for multi-tag classification. The maximum percentage of feature reduction was $95.93 \%$, which greatly reduced the computational complexity of the algorithm. 2) The wrapper model requires a specific mining algorithm and regards the performance as the evaluation criterion of the feature subsets. The model improves the performance of the mining algorithm, but the computational complexity tends to be higher relatively. For example, a method aiming to enhance the stability of sequence forward selection algorithm in the feature selection process was proposed in [5]. The experiment proved its effectiveness by employing the Bayesian classifier and obtained a better classification effect. In [6], Zhang Yudong et al. selected the binary PSO search method with mutation operator (MBPSO) to extract the primary features of the spam in the network. The experiment combined the search algorithm and C4.5 decision tree classifier, and the result proved that the method was more effective than other algorithms, such as ILS (Iterated Local Search) and GA (genetic algorithm). 3) The hybrid model takes advantage of the two models by adopting different evaluation criteria in different search stages. It selects the optimal candidate subset by independent measure method, and then uses the mining algorithm to obtain the final optimal subset, which ensures better performance of the mining algorithm and reduces the computational complexity of the search algorithm. As suggested in [7], a hybrid feature selection algorithm based on Maximum Minimum Backward Selection (MMBS) was proposed for liver tissue pathological image classification. The method took advantages of both the fast calculation of filter algorithm and the high classification accuracy of wrapper algorithm, and possessed better classification performance.

In order to obtain less computational complexity and higher accuracy, a new hybrid feature selection algorithm is proposed in this paper. As an independent measure method, the information gain method is used to calculate the information gain value of each feature. And the decision tree algorithm is regarded as a non-independent measure method and used to obtain the classification accuracy of the feature subset. Simultaneously, the process of subset generation is realized by Sequential Forward Floating Search (SFFS) method.

\section{Information Gain and SFFS}

\subsection{Information Gain}

Information Gain (IG) depends on the information entropy which the features provide to the system. Entropy is a parameter that characterizes the state of substance in thermodynamics and is a measure of the degree of chaos in the system. In 1948, C.E.Shannon, the father of information theory, applying entropy to the field of information, defined the average amount of information after redundancy was excluded as "information entropy"[8]. Information entropy is the quantitative measure of information and measures the degree of uncertainty of random variables[9].

Let $X$ be a random variable. If the degree of uncertainty of $X$ is higher, the greater amount of information is provided to the system. The information entropy of the random variable $X$ is defined as Eq.(1)[9]:

$$
H(X)=-\sum_{i} P\left(x_{i}\right) \log P\left(x_{i}\right)
$$


The information entropy of the random variable $Y$ with respect to the random variable $X$ is defined as Eq.(2) [9]:

$$
H(X \mid Y)=-\sum_{j} P\left(y_{i}\right) \sum_{i} P\left(x_{i} \mid y_{j}\right) \log P\left(x_{i} \mid y_{j}\right)
$$

Information Gain refers to the amount of information after eliminating the uncertainty, which is the difference of the information entropy between the random variable $X$ and the random variable $Y$ with respect to the random variable $X$. Therefore, information gain is shown in Eq.(3).

$$
I G(X, Y)=H(X)-H(X \mid Y)
$$

Information gain is a crucial concept in the information theory. For the classification system, information gain is in connection with each individual feature[10]. Information Gain is to measure the amount of information obtained for category prediction by knowing the presence or absence of a feature[11]. From Eq.(3) we can see that the information gain in the classification system is as Eq.(4):

$$
I G(t)=-\sum_{i=1}^{|C|} P\left(C_{i}\right) \log P\left(C_{i}\right)+P(t) \sum_{i=1}^{|C|} P\left(C_{i} \mid t\right) \log P\left(C_{i} \mid t\right)+P(\bar{t}) \sum_{i=1}^{|C|} P\left(C_{i} \mid \bar{t}\right) \log P\left(C_{i} \mid \bar{t}\right)
$$

Where $C_{i}$ means the category attribute, $P\left(C_{i}\right)$ is the probability of the $i$ th class value, $P(t)$ is the probability that feature $t$ occurs, and $P(\bar{t})$ is the probability that feature $t$ does not occur, $P\left(C_{i} \mid t\right)$ is the conditional probability that the class belongs to $C_{i}$ when the feature $t$ is included, while $P\left(C_{i} \mid \bar{t}\right)$ is the conditional probability that the class belongs to $C_{i}$ when the feature $t$ is not included.

The standard for measuring the importance of features is the amount of information that a feature brings to the classification system. The more the amount of information carries, the more important the feature is [11]. Therefore, when the feature selection is adopted in the filter model, some significant features of higher information gain are usually chosen to constitute the feature subset of the classification system, and the importance of the feature subset is improved.

\subsection{SFFS Algorithm}

Sequential Forward Floating Search Algorithm (SFFS) is an improved Sequential Forward Search (SFS) algorithm. SFS is a heuristic search approach. Setting the initial feature set is an empty set, a feature is added to the set at a time until the stop criterion is reached[12]. The process is described as follow: the initial feature set is $D\left(f_{1}, f_{2}, \ldots, f_{n}\right)$. Assuming that the feature subset $S_{k}$ contains $k$ features, the criterion function $A_{q}=A\left(S_{k}+s_{q}\right)$ needs to be calculated for each feature $s_{q}$ in set $\left\{D-s_{k}\right\}$. The feature that makes $A_{q}$ the largest is selected and added to $S_{k}$. The computational complexity of SFS algorithm is small, but it produces a nesting effect. Once a feature is selected to add to the feature subset, it can not be changed, which may lead to lower accuracy[13]. Therefore, SFFS is an improved algorithm aiming to overcome the shortcoming of SFS algorithm, which could remove features that are already added to the feature subset[14]. The method of adding features in SFFS algorithm is similar to SFS, however, SFFS algorithm could reduce the dimension of feature set by deleting the least significant feature during each iteration[15][16]. Suppose $k$ features $(k \leq n)$ have been selected into $S_{k}$, and SFFS algorithm mainly includes the following three steps [14]:

1) Using the basic SFS approach, find the feature $s_{k+1}$ in the set $\left\{D-S_{k}\right\}$ which maximizes the criterion function $A\left(S_{k}+S_{k+1}\right)$, and add it to $S_{k}$. Therefore, $S_{k+1}=S_{k}+s_{k+1}$;

2) Find the least significant feature in the set $S_{k+1}$. If $s_{k+1}$ is the least significant feature, i.e. $A\left(S_{k+1}-S_{k+1}\right) \geq A\left(S_{k+1}-s_{i}\right), 1 \leq i \leq k$. then set $k=k+1$ and return to step 1; Otherwise, exclude $s_{i}$ from $S_{k+1}$ to form a new feature set $S_{k}^{\prime}=S_{k+1}-s_{i}$. If $k=2$, then set $S_{k}=S_{k}^{\prime}, A\left(S_{k}\right)=A\left(S^{\prime}\right)$ and return to step 1, else return to step 3;

3) Find the least significant feature $S_{j}$ in the set $S_{k}^{\prime}$. If $A\left(S_{k}^{\prime}-S_{j}\right) \leq A\left(S_{k-1}\right)$, then set $S_{k}=S_{k}^{\prime}, A\left(S_{k}\right)=A\left(S_{k}^{\prime}\right)$ and return to step 1; Otherwise, exclude $S_{j}$ from $S_{k}^{\prime}$ to form a new feature set $S_{k-1}^{\prime}=S_{k}^{\prime}-S_{j}$, and set $k=k-1$. Now, if $k=2$, then set $S_{k}=S_{k}^{\prime}, A\left(S_{k}\right)=$ $A\left(S_{k}^{\prime}\right)$ and return to step 1, else repeat step 3.

The algorithm will stop iterating until one of the following conditions is satisfied [16]: 1) The value of the criterion function is 
no longer increased after a new feature is added; 2) The maximum number of features setted by the user has been reached.

\section{Hybrid Feature Selection Algorithm}

\subsection{The Main Idea of Hybrid Algorithm}

The sequential forward feature selection method is limited to some features with large contribution rate, however, in some practical cases, the optimal combination of features is usually not composed of these features[16]. Consequently, the feature subset obtained by the sequential forward feature selection method is likely to lead to lower classification accuracy. In order to reduce the number of iterations and improve the classification accuracy, we propose a new hybrid feature selection algorithm, which combines an independent measurement method based on information gain and a feature selection method based on Sequential Forward Floating Search and Decision Tree (DT). The main idea of the algorithm is shown in Figure 1.

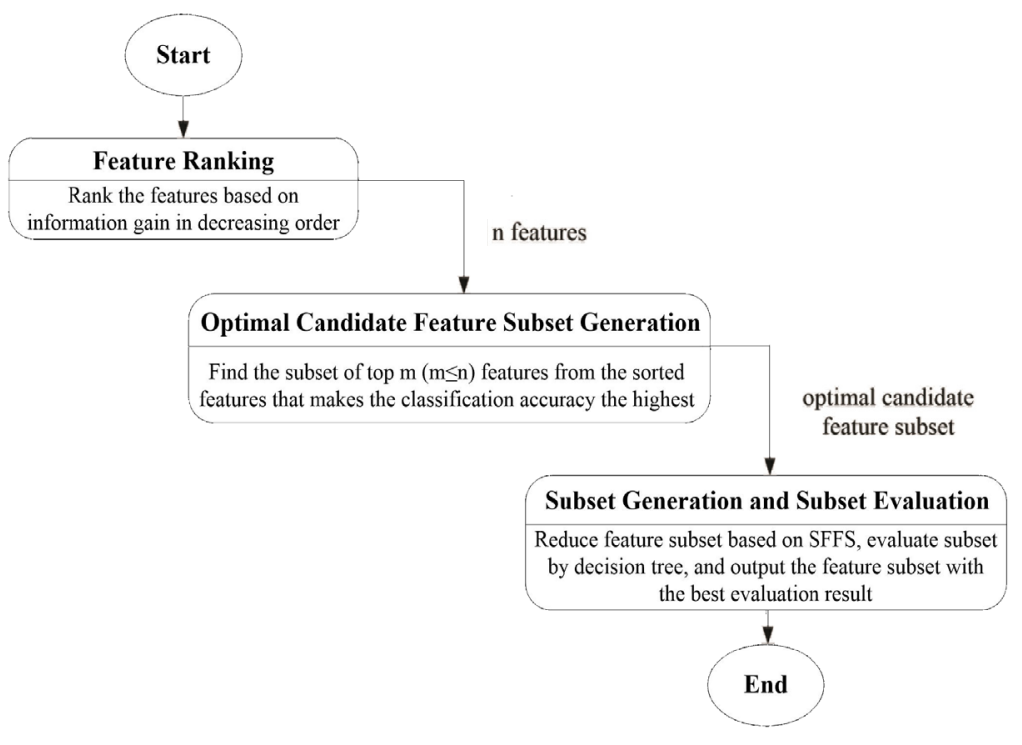

Figure 1. Main idea of hybrid algorithm

The hybrid algorithm is divided into three stages. Firstly, a filtering approach based on the information gain algorithm (IG) is employed in the feature ranking stage, aiming at calculating the information gain of features and ranking in decreasing order. Secondly, in the optimal candidate feature subset generation stage, the feature subset consisting of top $\mathrm{m}$ features is selected to make the classification accuracy the highest. The m-dimensional feature subset is the optimal candidate feature subset and is used as the input of the next stage. Finally, in the subset generation and subset evaluation stage, the optimal candidate feature subset obtained by the previous stage is iteratively generated new feature subsets by SFFS algorithm. Each iteration needs to be evaluated by the decision tree algorithm, and the subset with the best evaluation result is the final optimal feature subset selected by the hybrid feature selection algorithm.

\subsection{Hybrid Algorithm with Decision Tree}

The hybrid feature selection algorithm based on information gain and sequential forward floating search with decision tree is shown in Figure 2.

Set the initial feature set is $D\left(f_{1}, f_{2}, \ldots, f_{n}\right)$ and there are $n$ features $f_{i}$. Initialize set $S_{0}$ as an empty set. The candidate subset is $S_{\text {cand }}$, and the final feature subset selected by the hybrid algorithm is $S_{k}=\left\{s_{1}, s_{2}, \ldots, s_{k}\right)$, which contains $k$ features, $k \leq n$. There are two criterion functions $I G\left(s_{d}\right)$ and $A\left(S_{k}\right)$ in the hybrid algorithm. $I G\left(s_{d}\right)$ represents the information gain of features, and $I G\left(s_{i}\right)>I G\left(s_{j}\right)$ indicates that the feature $s_{i}$ has a greater information gain value than the feature $s_{j} ; A\left(S_{k}\right)$ means the classification accuracy obtained by the decision tree algorithm, and $A\left(S_{i}\right)>A\left(S_{j}\right)$ is true if the feature set $S_{i}$ perform better than the feature set $S_{j} . D \backslash s_{d}$ denotes that the feature set $D$ does not contain the feature $s_{d} A^{-}\left(s_{i}, S_{k}\right)$ represents the classification accuracy after removing the feature $s_{i}$ from the set $S_{k}$, Similarly, $\mathrm{A}^{+}\left(f_{i}, S_{k}\right)$ represents the classification accuracy after adding the feature $f_{i}$ to the set $S_{k}$, and 


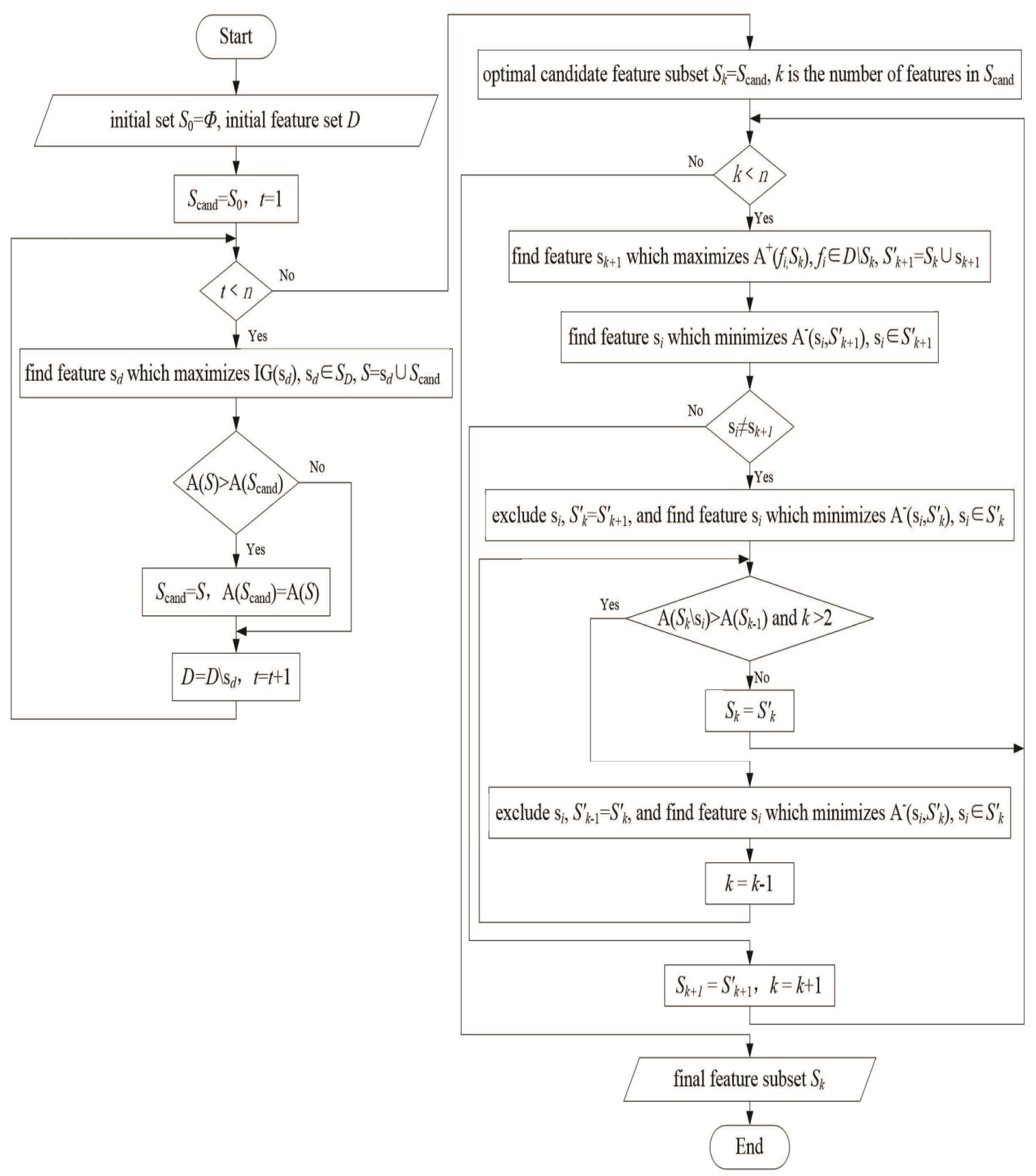

Figure 2. Hybrid feature selection algorithm with DT

$f_{i} \in D \backslash s_{k}$ denotes that $f_{i}$ belongs to the feature set in which $S_{k}$ is removed from $D$. The stop criterion of the hybrid algorithm is that the classification accuracy of the feature subset is no longer increased when a new feature is added. Eventually, the iteration is stopped and $S_{k}$ is output as the final optimal feature subset.

\section{Experiment and Result Analysis}

In order to verify the performance of the proposed algorithm, this paper compares the hybrid algorithm with a filter model based on information gain, a wrapper model based on SFFS and decision tree and another hybrid model based on SFFS and MC- 
SVM[17]. The number of reduced features, the number of iterations and the classification result of four algorithms are considered as three indexes to measure the performance of the algorithm. The experimental data sets are derived from UCI machine learning database[18]. Data sets need to be preprocessed before the experiment, and missing data is filled with mean or mode. Every data set is divided into training data $(80 \%)$ and test data $(20 \%)$. The final experimental results are obtained by ten fold cross validation.

\subsection{Number of Features}

Experiments are performed using 10 sets of data sets. The number of initial features, the nu mber of samples, and the number of features after reducing by four feature selection algorithms are shown in Table 1, where the hybrid-DT algorithm is the proposed hybrid algorithm with Decision Tree in section 2.2.

\begin{tabular}{|c|c|c|c|c|c|c|c|}
\hline & \multirow[b]{2}{*}{ Data Set } & \multirow{2}{*}{$\begin{array}{l}\text { Number of } \\
\text { initial fea- } \\
\text { tures }\end{array}$} & \multirow{2}{*}{$\begin{array}{l}\text { Number } \\
\text { of } \\
\text { samples }\end{array}$} & \multicolumn{4}{|c|}{ Number of features after reducing } \\
\hline & & & & Filter & Wrapper & $\begin{array}{c}\text { Hybrid } \\
\text {-DT }\end{array}$ & $\begin{array}{c}\text { Fybrid } \\
\text {-MC-SVM }\end{array}$ \\
\hline 1 & Diabetes & 9 & 768 & 3 & 4 & 5 & 1 \\
\hline 2 & Iris & 5 & 150 & 2 & 2 & 2 & 1 \\
\hline 3 & Glass & 10 & 214 & 7 & 3 & 4 & 8 \\
\hline 4 & Breast-cancer & 10 & 286 & 7 & 3 & 3 & 4 \\
\hline 5 & Hungarian & 14 & 294 & 2 & 1 & 1 & 5 \\
\hline 6 & Monks & 7 & 122 & 2 & 2 & 2 & 2 \\
\hline 7 & Heart-statlog & 14 & 270 & 3 & 3 & 3 & 3 \\
\hline 8 & Hepatitis & 20 & 155 & 6 & 2 & 2 & 5 \\
\hline 9 & Labor & 17 & 57 & 2 & 1 & 3 & 11 \\
\hline \multirow[t]{2}{*}{10} & Credit-g & 21 & 1000 & 4 & 3 & 5 & 8 \\
\hline & Mean & 12.7 & 331.6 & 3.8 & 2.4 & 3 & 4.8 \\
\hline
\end{tabular}

Table 1. Comparison of the number of features after reducing by four algorithms

As shown in Table 1, the number of features has a corresponding reduction after four algorithms are applied to 10 sets of data sets. The average number of features after reducing by hybrid algorithm with DT is less than filter algorithm and hybrid algorithm with MC-SVM, but slightly more than wrapper algorithm, and the mean in the hybrid algorithm with MC-SVM is the largest. The number of features after reducing by hybrid algorithm with MC-SVM is more than the hybrid algorithm with Decision Tree in most data sets. Monks and Heart-statlog have the same number of features after reducing by four algorithms. In Diabetes, Labor and Credit-g, the number of features after reducing by hybrid algorithm with DT is more than the first two algorithms, but in Glass, Breast-cancer, Hungarian, and Hepatitis, the number of features after reducing by hybrid algorithm with DT and wrapper algorithm is less than filter algorithm. The result shows that different feature selection algorithms applied to different data sets produce different effects. The maximum percentage of feature reduction in the hybrid algorithm with DT is $92.86 \%$, which greatly reduces the feature dimension and decreases the computational complexity for the subsequent classification and modeling.

\subsection{Number of Iterations and Classification Accuracy}

The number of iterations and classification accuracy are two important indexes to measure the performance of the feature selection algorithm. The number of iterations refers to the times of subset generated and evaluated during the feature selection process. And classification accuracy is the recognition rate obtained by 4.5 decision tree algorithm using 10 fold cross validation. The fewer the number of iterations and the higher the classification accuracy, the better the performance of the algorithm. Table 2 compares the proposed algorithm with the filter algorithm, the wrapper algorithm and the hybrid algorithm with MC-SVM. Experiments are performed using the same 10 sets of data sets as section 3.1. The number of iterations in the feature selection process and the classification accuracy obtained by C4.5 decision tree algorithm are calculated and shown in Table 2 .

As shown in Table 2, the number of iterations of filter algorithm is generally few, which indicates that the computational complexity of filter algorithm is low, however the classification accuracy of the feature subset selected by the filter algorithm is lower than

J8 Journal of Intelligent Computing Volume 9 Number 3 September 2018




\begin{tabular}{|l|l|l|l|l|l|l|l|c|}
\hline & \multicolumn{4}{|c|}{ Number of Iterations } & \multicolumn{5}{c|}{ Classification Accuracy } \\
\cline { 2 - 9 } & Filter & Wrapper & $\begin{array}{l}\text { Hybrid } \\
- \text { DT }\end{array}$ & $\begin{array}{l}\text { Hybrid } \\
\text {-MC-SVM }\end{array}$ & Hilter & Wrapper & $\begin{array}{l}\text { Hybrid } \\
\text {-DT }\end{array}$ & $\begin{array}{c}\text { Hybrid } \\
\text {-MC-SVM }\end{array}$ \\
\hline Diabetes & 8 & 31 & 28 & 30 & 74.6094 & 75.1302 & 75.9115 & 70.8333 \\
\hline Iris & 4 & 9 & 8 & 11 & 96.0000 & 96.0000 & 96.0000 & 96.0000 \\
\hline Glass & 9 & 33 & 36 & 34 & 69.6262 & 69.6262 & 74.2991 & 71.4953 \\
\hline Breast-cancer & 9 & 33 & 37 & 52 & 75.5245 & 75.8741 & 75.8741 & 76.5734 \\
\hline Hungarian & 13 & 25 & 38 & 118 & 81.2925 & 81.2925 & 81.2925 & 84.6939 \\
\hline Monks & 6 & 15 & 12 & 12 & 93.4426 & 93.4426 & 93.4426 & 93.4426 \\
\hline Heart-statlog & 13 & 49 & 25 & 26 & 85.1852 & 85.1852 & 85.1852 & 85.1852 \\
\hline Hepatitis & 19 & 56 & 65 & 73 & 83.8710 & 85.1613 & 85.1613 & 87.7419 \\
\hline Labor & 16 & 31 & 40 & 96 & 78.9474 & 80.7018 & 89.4737 & 89.4737 \\
\hline Credit-g & 20 & 77 & 53 & 125 & 74.6000 & 73.0000 & 76.7000 & 76.1000 \\
\hline Mean & 11.7 & 35.9 & 34.2 & 56.2 & 81.3099 & 81.5414 & 83.3340 & 83.1539 \\
\hline Standard deviation & 5.1390 & 18.9971 & 16.3205 & 40.6228 & 8.0626 & 8.0618 & 7.4001 & 8.4803 \\
\hline
\end{tabular}

Table 2. Number of iterations and classification accuracy

other algorithms broadly. The number of iterations and classification accuracy of wrapper algorithm are higher than the filter algorithm. The hybrid algorithm with DT has slightly more iterations than filter algorithm, but the classification accuracy of hybrid algorithm with DT is higher in most data sets and the maximum accuracy is improved by about $10.5 \%$. Compared with the wrapper algorithm, the hybrid algorithm with DT possesses higher classification accuracy on the basis of maintaining less iterations in Diabetes and Credit-g; In Iris, Monks and Heart-statlog, two algorithms have the same accuracy, but the number of iterations of the hybrid algorithm with DT is significantly reduced and the maximum decline is about 49\%; And in Glass and Labor, the number of iterations is slightly more, but the classification accuracy of the hybrid algorithm with DT is significantly improved and the maximum increase is about $8.8 \%$, indicating that the overall performance of the hybrid algorithm with DT is better than the filter algorithm and the wrapper algorithm.

Compared with the hybrid algorithm with MC-SVM, the average number of iterations of the hybrid algorithm with DT is not only less, but the classification accuracy is higher. In Diabetes and Credit-g, the hybrid algorithm with DT possesses higher classification accuracy on the basis of maintaining less iterations and the maximum decline of iterations is $57.6 \%$; In Iris, Heart-statlog and Labor, two algorithms have the same accuracy, but the number of iterations of the hybrid algorithm with DT is significantly reduced and the maximum decline is about 58.3\%; In Breast-cancer, Hungarian and Hepatitis, the classification accuracy of the hybrid algorithm with DT is slightly lower, but the number of iterations is significantly decreased and the maximum decline is about 67.8\%; And in Glass, the number of iterations is slightly more, but the classification accuracy of the hybrid algorithm with DT is improved. The above analyses show that the computational complexity of the hybrid algorithm with DT is generally lower than the hybrid algorithm with MC-SVM, indicating that the overall performance of the hybrid algorithm with DT is better than the hybrid algorithm with MC-SVM.

We can see from the mean that the computational complexity of wrapper algorithm is nearly three times than filter algorithm and the accuracy of wrapper algorithm is improved by about $0.23 \%$. However, compared with the wrapper algorithm, the hybrid algorithm with DT is computationally cheaper and the accuracy is improved by about $2.02 \%$, which is nearly 9 times than the former, indicating that the hybrid algorithm with DT performs better. In addition, the standard deviation shows that the fluctuation of classification accuracy obtained by the hybrid algorithm with DT in different data sets is the smallest, which indicates that the stability of the hybrid algorithm with DT is better. To sum up, The hybrid algorithm with DT combines the advantages of filter algorithm and wrapper algorithm, possessing higher classification accuracy in the case of ensuring less iterations, and is proved to be a good algorithm in the field of classification. 


\section{Conclusion}

In this paper, a Filter-Wrapper hybrid feature selection algorithm based on information gain and SFFS with Decision Tree is proposed. The experiments prove that the hybrid algorithm improves the computational efficiency of feature selection process and obtains higher classification accuracy. Combining the filter algorithm with the wrapper algorithm, the candidate feature subset is acquired by using the independent measure method based on information gain, and then the SFFS search method and the decision tree mining algorithm are employed to obtain the final optimal feature subset. The hybrid algorithm takes advantages of both the fast calculation of filter algorithm and the high classification accuracy of wrapper algorithm, and is proved to be a feature selection algorithm with better performance. However, the method does not take fully into account the correlation among features. Therefore, it is worthy to study how to improve the correlation of features by combining other correlation measurement methods in the case of maintaining high accuracy.

\section{References}

[1] Sankar, K. V., Uma, D. S., Subin, P. S., et al. (2014). A literature review on DataMining, International Journal of Research in Computer Applications \& Robotics, 2 (7) 95-101.

[2] Liu, H., Yu, L. (2005). Toward integrating feature selection algorithm for classification and clustering, IEEE Transactions on Knowledge \& Data Engineering, 17 (4) 491-502.

[3] Dash, M., Liu, H. (1997). Feature selection for classification, Intelligent Data Analysis, 1, (3)131-156.

[4] Spolaôr, N., Cherman, E. A., Monard, M. C., et al. (2012). Filter approach feature selection methods to support multi-label learning based on ReliefF and information gain, Lecture Notes in Computer Science, p. 72-81.

[5] Cateni, S., Colla, V. (2015). Improving the stability of sequential forward variables selection, In: $15^{\text {th }}$ International Conference on Intelligent Systems Design and Applications Isda, p 488-962.

[6] Zhang, Y., Wang, S., Phillips, P., et al. (2014). Binary PSO with mutation operator for feature selection using decision tree applied to spam detection, Knowledge-Based Systems, 64, p. 22-31.

[7] Liu, H., Jiang, H., Zheng, R. (2016). The hybrid feature selection algorithm based on maximum minimum backward selection search strategy for liver tissue pathological image classification, Computational \& Mathematical Methods in Medicine, 23, p. 19.

[8] Shannon, C. E. (1948). A mathematical theory of communication, Bell System Technical Journal, 27, p. 379-423, 623-656.

[9] Tang, Z., Lu, Z., Jiang, B., et al. (2013). Entropy-Based importance measure for uncertain model inputs, Aiaa Journal, 51 (10) p. 2319-2334.

[10] Ong, B. Y., Goh, S. W., Xu, C. (2015). Sparsity adjusted information gain for feature selection in sentiment analysis, IEEE International Conference on Big Data, p 2122-2128.

[11] Xu, J., Jiang, H. (2015). An improved information gain feature selection algorithm for SVM text classifier, International Conference on Cyber-Enabled Distributed Computing and Knowledge Discovery, p. 273-276.

[12] Liogien, T., Tamulevi ius, G. (2015). SFS feature selection technique for multistage emotion recognition, IEEE Information, Electronic and Electrical Engineering, p.1-4, 2015.

[13] Liogien, T., Tamulevi ius, G. (2017). Multi-Stage recognition of speech emotion using sequential forward feature selection, Electrical Control \& Communication Engineering, 10, p. 35-41.

[14] Pudil, P., Novovi ová, J., Kittler, J. (1994). Floating search methods in feature selection, Pattern Recognition Letters, 15 (11) p.1119-1125.

[15] Schenk, J., Kaiser, M., Rigoll, G. (2009). Selecting features in on-line handwritten whiteboard note recognition:SFS or SFFS, IEEE. International Conference on Document Analysis and Recognition, p.1251-1254.

[16] Baker, M. C., Kerr, A.S., Hames, E., et al. (2012). An SFFS technique for EEG feature classification to identify sub-groups, International Symposium on Computer-Based Medical Systems, p.1-4.

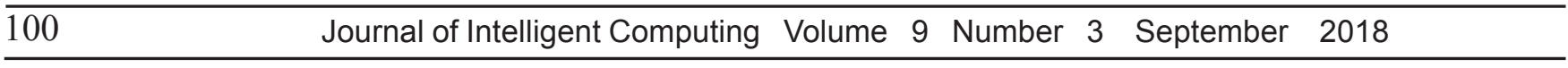


[17] Rajalakshmi, N., Lakshmi, P. V. (2015). MRI brain image classification—a hybrid approach, International Journal of Imaging Systems \& Technology, 25 (3) 226-244.

[18] Frank, A., Asuncion, A. (2011). UCIrvine machine learning repository, http://archive.ics.uci.edu/ml.

DING Jianli, was born in 1963. He received his PhD degree from Nankai University, China. Now he is a Professor in Civil aviation University of China, Tianjin, China. His research interests include intelligent information processing, big data mining in civil aviation, intelligent algorithm and application of civil aviation. 